\title{
Centriole biogenesis: a tale of two pathways
}

\author{
Jadranka Loncarek, Greenfield Sluder, and Alexey Khodjakov \\ Jadranka Loncarek and Alexey Khodjakov are in the Division of Molecular Medicine, Wadsworth \\ Center, Albany NY 12201-0509, USA. Greenfield Sluder is in the Department of Cell Biology, \\ University of Massachusetts Medical School, Worcester MA 01605, USA.
}

\section{Abstract}

Two recent studies in Drosophila demonstrate that overexpression of proteins required for centriole duplication can not only induce centriole over-duplication in cells containing centrioles, but can also drive de novo centriole assembly in unfertilized eggs that initially lack centrioles. These studies offer a new perspective on the mechanisms that control centriole duplication.

The animal cell's primary microtubule-organizing centre, the centrosome, contains a pair of centrioles surrounded by pericentriolar material (PCM). As the number of centriole pairs determines the number of centrosomes the cell contains, the assembly of new centrioles must be under tight temporal and numerical control. At the time of DNA synthesis, each centriole duplicates to produce a mother and daughter centriole pair, which form two centrosomes to organize the mitotic spindle poles. If more than two centrosomes are present in the cell at the onset of mitosis, the cell often assembles a multipolar mitotic spindle. This inevitably leads to unequal chromosome distribution and consequent aneuploidy and genomic instability, both hallmarks of aggressive tumour cells ${ }^{1}$. The rules of centriole duplication seem to be quite simple: each mother centriole produces just one daughter, and it does so only once per cell cycle. However, the molecular mechanisms that govern centriole duplication have been an enduring mystery. Two recent reports 2,3 shed new light on this subject and raise a number of fascinating questions.

Rodrigues-Martin et al. ${ }^{2}$ overexpressed Sak/Plk4, a kinase known to be a positive regulator of normal centriole duplication 4,5 , in Drosophila embryos and unfertilized eggs. In early syncytial-stage embryos, increased levels of Plk4 caused the amplification of centrioles that seemed to arise from the reduplication of the sperm centrioles. Importantly, overexpression of Plk4 in unfertilized eggs, which have no preexisting centrioles because they are naturally lost during oogenesis, induced a rapid de novo assembly of multiple centrioles throughout the cytoplasm. The de novo-assembled centrioles were able to organize functional centrosomes and underwent subsequent duplication. After this initial burst of de novo centriole formation, it appears that Plk4-overexpressing oocytes suppressed this pathway and completely switched to repetitive rounds of centriole duplication ${ }^{2}$.

Peel et al. ${ }^{3}$ provided similar Plk4 data and additionally demonstrated that the de novo formation of centrioles in the unfertilized egg can also be induced by overexpression of either of the two centriolar proteins, Sas-6 or Sas-4. During centriole duplication, Sas-6 and Sas-4 are sequentially recruited to the forming centriole in a Plk4-dependent fashion ${ }^{6}$, and are essential for the formation of the central tube and subsequent assembly of centriolar microtubules respectively ${ }^{7}$. Intriguingly, Peel et al. also demonstrated that overexpression of Sak/Plk4 or 
Sas-6, but not Sas-4, induced accumulation of extra centrioles in some somatic tissues (for example, the brain), but not in the male or female germlines. In tissues overexpressing Sas-6, the supernumerary centrioles arose from more than one round of duplication within a single cell cycle.

There are some fascinating, and perhaps not fully resolved, issues raised by these two studies. One value of the studies is the new insight they provide into the relationship between the two modes of centriole biogenesis observed in nature: the duplication of pre-existing maternal centrioles, and the de novo assembly of centrioles without a pre-existing mother centriole.

Centriole reproduction in somatic cells is based on the duplication of mature 'mother' centrioles. A daughter centriole forms in close spatial association with the wall of the mother centriole, suggesting that the mother provides a singular site or 'template' that seeds growth. Mother and daughter centrioles subsequently remain attached or 'engaged' until the late steps of mitosis ${ }^{8}$. An obvious question is why the assembly of only one daughter centriole occurs just once per cell cycle. Recent work has lent support to the proposal that the retained attachment of the daughter centriole to the mother prevents formation of additional progeny until the daughter and mother separate at anaphase onset due to the action of the proteolytic enzyme separase, which also causes chromosome disengagement ${ }^{8}$. During the next cell cycle, the actual initiation of centriole duplication occurs only when cytoplasmic conditions become permissive during S phase.

In addition, and seemingly paradoxically, longstanding observations demonstrate that new centrioles can assemble without a preexisting centriole. A classic example of this process comes from mouse development, where both maternal and paternal centrioles are eliminated during gametogenesis. Although the zygote initially has no centrioles, by the 16-32-blastomere stage of development, each cell regains these organelles in proper copy number ${ }^{9}$. In recent years, it has also become clear that de novo assembly exists in a variety of somatic cells that normally rely on canonical template duplication 10,11 . In somatic cells, the de novo pathway becomes active only if the resident centrioles are lost or experimentally removed from the cell. The presence of a single resident centriole is sufficient to completely suppress the de novo pathway in somatic cells ${ }^{11}$. When the de novo pathways becomes active, it results in the formation of a variable number of centrioles (between 1 and 14) ${ }^{11}$.

Whether these two seemingly disparate modes of centriole biogenesis are fundamentally different or represent variations of a common pathway remains to be determined. The Rodrigues-Martins et al. and Peel et al. reports reveal that de novo assembly can be triggered by individual overexpression of proteins involved in templated biogenesis (for example, Sak/ Plk4 or Sas-6), thus favouring the latter hypothesis and raising a series of issues. Although overexpression of Plk4 activates de novo assembly in unfertilized eggs that lack centrioles, this pathway remains suppressed in centriole-containing cells when this kinase is overexpressed (Fig. 1). This inhibition could be explained by a putative difference in concentrations of regulatory components between the centrosome and the cytoplasm. However, in this case, highlevel expression of Sak/Plk4 or Sas6 should result in a cytoplasmic concentration sufficient to support de novo assembly, which does not occur in the presence of a resident centrosome. What blocks the de novo assembly under these conditions? There must be an additional regulatory mechanism - perhaps based on an activity located at the resident centrosome. Another issue concerns the manner in which the unfertilized egg suppresses de novo centriole assembly, even though it contains subunit pools sufficient to make $1 \times 10^{13}$ centriole pairs. As individual overexpression of either Sas4/Plk4, Sas-6 or Sas-4 triggers de novo assembly in unfertilized eggs, this again implies that inhibition of this pathway is not due to the shortage of a particular protein, but instead to the existence of an additional centriole-independent regulatory 
mechanism. Although the nature of this mechanism remains a mystery, the suggestion that it exists represents a significant step forward.

Additionally, these studies provide new insights into the two different aspects of centriole duplication that must be controlled to prevent overproduction of daughter centrioles: first, what limits the number of daughters that form in association with a single mother to just one; and second, how are repetitive duplications of the same mother prevented within a single cell cycle? Overexpression of Plk4 in mammalian culture cells has been shown to induce formation of multiple centriolar precursors surrounding the mother centriole ${ }^{5}$ that ultimately mature into morphologically complete centrioles 12 - an effect that requires Plk4 activity at the centriole 5 . This suggests that localized phosphorylation of a 'seed' on the wall of the mother centriole can initiate the growth of the daughter centriole. Excessive activity of Plk4 results in phosphorylation of multiple seeds and thus formation of multiple daughter centrioles ${ }^{13}$. Similar effects could be expected for Sas-6, whose recruitment to the centriole is Plk4dependent ${ }^{7,14}$. In contrast to the effects observed in mammalian cells, Rodrigues-Martins et al. and Peel et al. demonstrate that overexpression of Sak/Plk4 in Drosophila embryo and somatic cells does not induce simultaneous formation of multiple daughter centrioles. Instead, it seems to lead to repeated centriole duplication within one cell cycle. The same phenomenon was observed in live Drosophila embryos overexpressing Sas-6 (ref. 3). Thus, the manner in which Plk4 and Sas6 influence the duplication processes for centrioles seems to be different between cells of different organisms (Fig. 2).

Furthermore, the extent of centriole overduplication when Sak/Plk4 or Sas-6 are overexpressed varies between different Drosophila tissues: for example, when identical levels of these proteins are expressed, supernumerary centrioles readily accumulated in brain somatic cells and in unfertilized eggs, but not in spermatocytes or immature eggs undergoing oogenesis ${ }^{3}$. This suggests that centriole duplication is subject to additional controls that are cell-type specific. In this context, overproduction of daughter centrioles in human cells overexpressing Plk4 was enhanced if cells also overexpressed cyclin E/Cdk2 (ref. 12) or the HPV-16 E7 oncoprotein, which constitutively increases the level of cyclin $\mathrm{E}^{12}$. Thus, even moderate elevation of Plk4 levels in cells, coupled with higher than usual expression of proteins and kinases involved in cell-cycle regulation, can lead to centriole amplification and genomic instability.

Although the details of how Sak/Plk4, Sas6 and Sas4 participate in the formation of centrioles remain obscure, the Rodrigues-Martins et al. and Peel et al. studies demonstrate that these proteins are key players in both canonical duplication and de novo assembly of centrioles. Perhaps these two seemingly disparate pathways for centriole biogenesis are not fundamentally different: canonical centriole duplication might represent de novo assembly spatially restricted to the immediate proximity of mother centrioles inside the cloud of PCM. In the absence of resident centrioles small pieces of PCM would be spread throughout the cytoplasm providing a variable number of transient 'shelters' in which assembly of centrioles can, in principle, be initiated. In the presence of resident centrioles, these pieces of PCM are efficiently transported to the centrosome ${ }^{15}$, thus spatially restricting the environment in which new centrioles can form. This speculative scenario suggests a way in which the overexpression of Sak/Plk4 or Sas-6, if they were involved in the formation or stabilization of these shelters, would promote overduplication in centrioles containing cells and also de novo formation in cells lacking resident centrioles.

\section{References}

1. Nigg EA. International Journal of Cancer 2006;119:2717-2723. 
2. Rodrigues-Martins A, Riparbelli M, Callaini G, Glover DM, Bettencourt-Dias M. Science 2007;316:1046-1050. [PubMed: 17463247]

3. Peel N, Stevens NR, Basto R, Raff JW. Curr. Biol 2007;17:834-843. [PubMed: 17475495]

4. Bettencourt-Dias M, et al. Curr. Biol 2005;15:2199-2207. [PubMed: 16326102]

5. Habedank R, Stierhof YD, Wilkinson CJ, Nigg EA. Nature Cell Biol 2005;7:1140-1146. [PubMed: 16244668]

6. Delattre M, Canard C, Gonczy P. Curr. Biol 2006;16:1844-1849. [PubMed: 16979563]

7. Pelletier L, O‘Toole E, Schwager A, Hyman AA, Muller-Reichert T. Nature 2006;444:619-623. [PubMed: 17136092]

8. Tsou MF, Stearns T. Nature 2006;442:947-951. [PubMed: 16862117]

9. Szollosi D, Calarco P, Donahue RP. J. Cell Sci 1972;11:521-541. [PubMed: 5076360]

10. Uetake Y, et al. J. Cell Biol 2007;176:173-182. [PubMed: 17227892]

11. La Terra S, et al. J. Cell Biol 2005;168:713-720. [PubMed: 15738265]

12. Duensing A, et al. Oncogene. 2007

13. Nigg EA. Trends Cell Biol 2007;17:215-221. [PubMed: 17383880]

14. Dammermann A, et al. Dev. Cell 2004;7:815-829. [PubMed: 15572125]

15. Young A, Dictenberg JB, Purohit A, Tuft R, Doxsey SJ. Mol. Biol. Cell 2000;11:2047-2056. [PubMed: 10848628] 

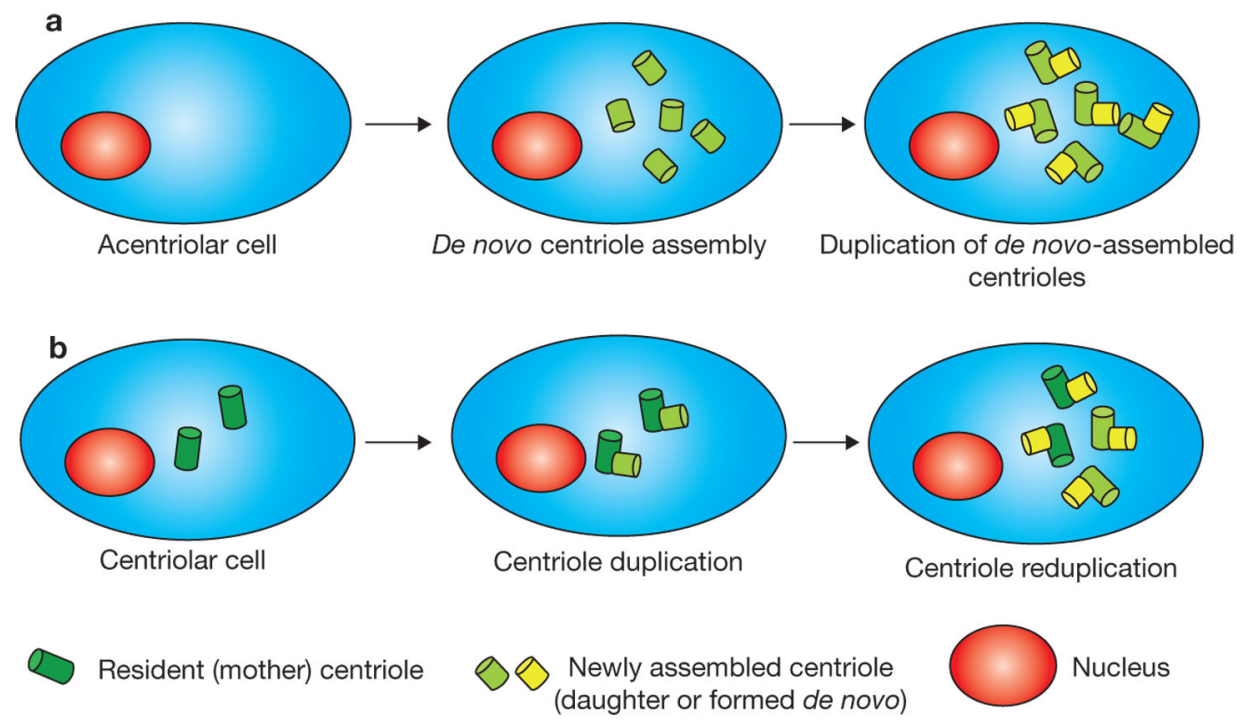

Figure 1. Effects of Sak/Plk4 overexpression in different cell types

(a) In unfertilized Drosophila eggs, or in cultured cells that lack resident centrioles, overexpression of Sak/Plk4 induces de novo assembly of centrioles. Subsequently, these centrioles duplicate. (b) In contrast, when Sak/Plk4 is overexpressed in centriolar cells the de novo pathway is not activated, although the resident centrioles overduplicate. 
a U2-OS cells

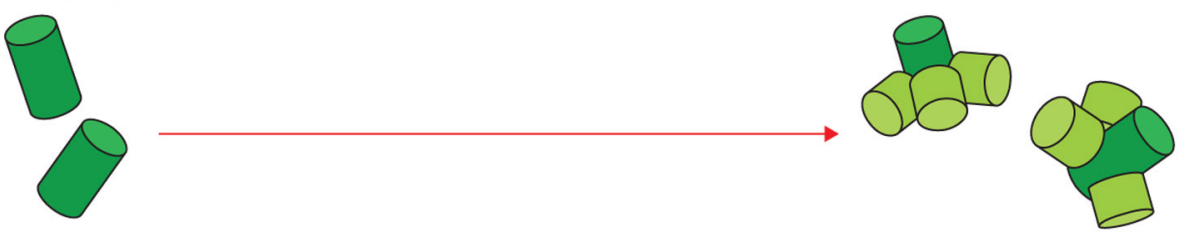

b Drosophila cells

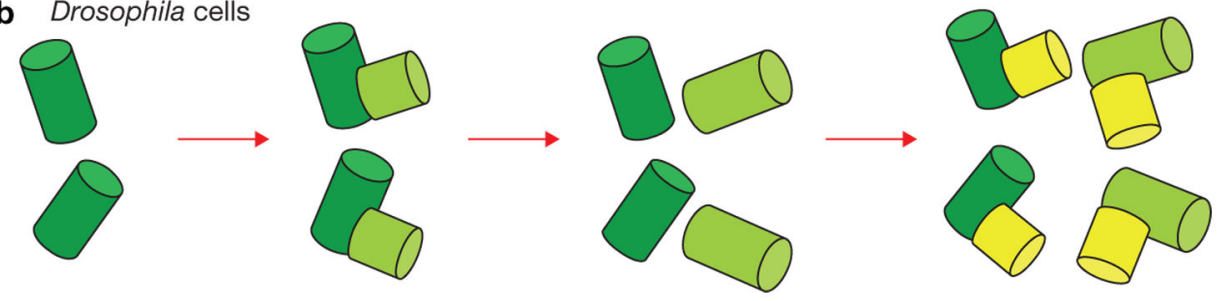

Figure 2. Mechanisms of centriole reduplication when Sak/Plk4 or Sas6 are overexpressed (a) In human cultured cells (U2-OS), centriole overexpression of Plk4 induces formation of multiple daughters in association with a single mother centriole. (b) In Drosophila embryos and somatic brain cells, a single daughter centriole forms in association with each resident mother. However, the centriolar cycle is accelerated when either Sas-6 or Sak/Plk4 are overexpressed, so that each mother centriole duplicates multiple times in a single cell cycle. 